\title{
Algorithmic Approach to the Split Problems
}

\author{
Ming Ma \\ Tianjin and Education Ministry, Key Laboratory of Advanced Composite Materials, Tianjin 300387, China
}

Correspondence should be addressed to Ming Ma; maming@tjpu.edu.cn

Received 6 June 2013; Accepted 26 June 2013

Academic Editor: Abdellah Bnouhachem

Copyright (C) 2013 Ming Ma. This is an open access article distributed under the Creative Commons Attribution License, which permits unrestricted use, distribution, and reproduction in any medium, provided the original work is properly cited.

This paper deals with design algorithms for the split variational inequality and equilibrium problems. Strong convergence theorems are demonstrated.

\section{Introduction}

Let $\mathbb{Q}$ be a real Hilbert space. Let $\mathbb{C}$ and $\mathbb{Q}$ be two nonempty closed convex subsets of $\mathbb{W}$. Consider the following problem.

Problem 1. Find a point $u^{\S} \in \mathbb{C}$ such that

$$
\Psi\left(u^{\S}\right) \in \mathbb{Q}
$$

This problem is called split feasibility problem when $\Psi$ is a bounded linear operator. In this case, Problem 1 can be applied to many practical problems such as signal processing and image reconstruction. Specifically, we can find the prototype of Problem 1 in intensity-modulated radiation therapy; see, for example, [1-3]. Based on this relation, many mathematicians were devoted to study the split feasibility problem and develop its iterative algorithms. Related works can be found in [4-8] and the references therein.

Let $\mathbb{A}, \Psi: \mathbb{C} \rightarrow \mathbb{U}$ be two mappings. Consider the variational inequality of finding $u^{\dagger} \in \mathbb{C}, \Psi\left(u^{\dagger}\right) \in \mathbb{C}$ such that

$$
\left\langle A u^{\dagger}, \Psi(u)-\Psi\left(u^{\dagger}\right)\right\rangle \geq 0,
$$

for all $\Psi(u) \in \mathbb{C}$. We use $\operatorname{VI}(\mathbb{A}, \Psi)$ to denote the set of solutions of (2). Variational inequality problems have important applications in many fields such as elasticity, optimization, economics, transportation, and structural analysis, and various numerical methods have been studied by many researchers; see, for instance, [9-17].
Let $\varrho: \mathbb{C} \times \mathbb{C} \rightarrow \mathbb{R}$ be an equilibrium bifunction; that is, $\varrho(u, u)=0$ for each $u \in \mathbb{C}$. Consider the equilibrium problem which is to find $u^{*} \in \mathbb{C}$ such that

$$
\varrho\left(u^{*}, v\right) \geq 0, \quad \forall v \in \mathbb{C} .
$$

Denote the set of solutions of (3) by $\operatorname{EP}(\varrho, \mathbb{C})$. The equilibrium problems include fixed point problems, optimization problems, and variational inequality problems as special cases. Some algorithms have been proposed to solve the equilibrium problems; see, for example, [18-22]. Thus it is an interesting topic associated with algorithmic approach to the variational inequality and equilibrium problems. In this paper, our main purpose is to study the following split problem involved in the variational inequality and equilibrium problems. Find a point $x^{\natural}$ such that

$$
\begin{gathered}
x^{\natural} \in \mathrm{VI}(\mathbb{A}, \Psi), \\
\Psi\left(x^{\natural}\right) \in \operatorname{EP}(\varrho, \mathbb{C}) .
\end{gathered}
$$

We are devoted to study (4) with operator $\Psi$ being a nonlinear mapping. For this purpose, we develop an iterative algorithm for solving the split problem (4). We can compute $x^{\natural}$ iteratively by using our algorithm. Convergence analysis is given under some mild assumptions.

\section{Basic Concepts}

Let $\mathbb{C}$ be a nonempty closed convex subset of a real Hilbert space $\mathbb{U}$. An operator $\mathbb{B}: \mathbb{C} \rightarrow \mathbb{W}$ is said to be

(i) monotone $\rightarrow\langle u-v, \mathbb{B} u-\mathbb{B} v\rangle \geq 0$ for all $u, v \in \mathbb{C}$; 
(ii) strongly monotone $\rightarrow\langle u-v, \mathbb{B} u-\mathbb{B} v\rangle \geq \zeta\|u-v\|^{2}$ for some constant $\zeta>0$ and for all $u, v \in \mathbb{C}$;

(iii) inverse-strongly monotone $\rightarrow\langle u-v, \mathbb{B} u-\mathbb{B} v\rangle \geq$ $\varsigma\|\mathbb{B} u-\mathbb{B} v\|^{2}$ for some $\varsigma>0$ and for all $u, v \in \mathbb{C}$; in this case, $\mathbb{B}$ is called $\varsigma$-inverse strongly monotone;

(iv) $\varsigma$-inverse strongly $\theta$-monotone $\rightarrow\langle\theta(u)-\theta(v), \mathbb{B} u-$ $\mathbb{B} v\rangle \geq \varsigma\|\mathbb{B} u-\mathbb{B} v\|^{2}$ for all $u, v \in \mathbb{C}$ and for some $\varsigma>0$, where $\theta: \mathbb{C} \rightarrow \mathbb{C}$ is a mapping.

A mapping $\vartheta: \mathbb{C} \rightarrow \mathbb{H}$ is said to be

(i) nonexpansive $\rightarrow\|\vartheta u-\vartheta v\| \leq\|u-v\|$ for all $u, v \in \mathbb{C}$;

(ii) firmly nonexpansive $\rightarrow\|\vartheta u-\vartheta v\|^{2} \leq\langle u-v, \vartheta u-\vartheta v\rangle$ for all $u, v \in \mathbb{C}$;

(iii) $L$-Lipschitz continuous $\rightarrow\|\vartheta u-\vartheta v\| \leq L\|u-v\|$ for some constant $L>0$ and for all $u, v \in \mathbb{C}$. In such a case, $\vartheta$ is said to be $L$-Lipschitz continuous.

In the sequel, we use $\operatorname{Fix}(\vartheta)$ to denote the set of fixed points of $\vartheta$.

Let $\mathbb{A}: \mathbb{H} \rightarrow 2^{\mathbb{\sharp}}$ be a multivalued mapping. The effective domain of $\mathbb{A}$ is denoted by $\operatorname{dom}(\mathbb{A})$. $\mathbb{A}$ is said to be

(i) monotone $\rightarrow\langle x-y, u-v\rangle \geq 0$ for all $x, y \in \operatorname{dom}(\mathbb{A})$, $u \in \mathbb{A} x$, and $v \in \mathbb{A} y$;

(ii) maximal monotone $\rightarrow \mathbb{A}$ is monotone and its graph is not strictly contained in the graph of any other monotone operator on $\mathbb{H}$.

A function $f: \mathbb{W} \rightarrow \mathbb{R}$ is said to be convex if for any $u, v \in$ $\mathbb{M}$ and for any $\tau \in[0,1], f(\tau u+(1-\tau) v) \leq \tau f(u)+(1-\tau) f(v)$.

Let $\operatorname{proj}_{\mathbb{C}}: \mathbb{C} \rightarrow \mathbb{W}$ be the metric projection from $\mathbb{H}$ onto $\mathbb{C}$. It is known that $\operatorname{proj}_{\mathbb{C}}$ satisfies the following inequality:

$$
\left\langle x-\operatorname{proj}_{\mathbb{C}} x, y-\operatorname{proj}_{\mathbb{C}} x\right\rangle \leq 0 .
$$

for all $x \in \mathbb{H}$ and $y \in \mathbb{C}$. From this characteristic inequality, we can deduce that proj $_{\mathbb{C}}$ is firmly nonexpansive.

\section{Useful Lemmas}

In this section, we present several lemmas which will be used in the next section.

Lemma 2 (see [19]). Let $\mathbb{C}$ be a nonempty closed convex subset of a real Hilbert space $\mathbb{H}$. Let $\varrho: \mathbb{C} \times \mathbb{C} \rightarrow \mathbb{R}$ be a bifunction. Assume that $\varrho$ satisfies the following conditions:

(1) $\varrho(u, u)=0$ for all $u \in \mathbb{C}$;

(⿰2) $\varrho$ is monotone, that is, $\varrho(u, v)+\varrho(v, u) \leq 0$ for all $u, v \in \mathbb{C}$;

(⿰3) for each $u, v, w \in \mathbb{C}, \lim _{t \downarrow 0} \varrho(t w+(1-t) u, v) \leq$ $\varrho(u, v)$;

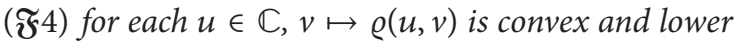
semicontinuous.

Let $\omega>0$ and $u \in \mathbb{C}$. Then there exists $w \in \mathbb{C}$ such that

$$
\varrho(w, v)+\frac{1}{\omega}\langle v-w, w-u\rangle \geq 0, \quad \forall v \in \mathbb{C} .
$$

Set $F_{\Phi}(u)=\{w \in \mathbb{C}: \varrho(w, v)+(1 / \omega)\langle v-w, w-u\rangle \geq 0$ for all $v \in \mathbb{C}\}$. Then one have the following:

(i) $F_{\varrho}$ is single valued and $F_{\varrho}$ is firmly nonexpansive,

(ii) $\operatorname{EP}(\varrho, \mathbb{C})$ is closed and convex and $\operatorname{EP}(\varrho, \mathbb{C})=\operatorname{Fix}\left(F_{\omega}\right)$.

Lemma 3 (see [23]). Let $\mathbb{C}$ be a nonempty closed convex subset of a real Hilbert space $\mathbb{H}$. For $x \in \mathbb{H}$, let the mapping $F_{\omega}$ be the same as in Lemma 2. Then for $\mu, v>0$ and $x \in \mathbb{U}$, one has

$$
\left\|F_{\mu}(x)-F_{\nu}(x)\right\|^{2} \leq \frac{\mu-\nu}{\mu}\left\langle F_{\mu}(x)-F_{\nu}(x), F_{\mu}(x)-x\right\rangle .
$$

Lemma 4 (see [24]). Let $\left\{u_{n}\right\}$ and $\left\{v_{n}\right\}$ be two bounded sequences in a Banach space $\mathbb{E}$, and let $\left\{\kappa_{n}\right\}$ be a sequence in $[0,1]$ satisfying $0<\liminf _{n \rightarrow \infty} \kappa_{n} \leq \limsup _{n \rightarrow \infty} \kappa_{n}<$ 1. Suppose $u_{n+1}=\left(1-\kappa_{n}\right) v_{n}+\kappa_{n} u_{n}$ for all $n \geq 0$ and $\limsup _{n \rightarrow \infty}\left(\left\|v_{n+1}-v_{n}\right\|-\left\|u_{n+1}-u_{n}\right\|\right) \leq 0$. Then, $\lim _{n \rightarrow \infty}\left\|u_{n}-v_{n}\right\|=0$.

Lemma 5 (see [25]). Let $\mathbb{C}$ be a nonempty closed convex subset of a real Hilbert space $\mathbb{H}$. Let $\mathbb{S}: \mathbb{C} \rightarrow \mathbb{C}$ be a nonexpansive mapping with $\operatorname{Fix}(\mathbb{S}) \neq \emptyset$. Then $\mathbb{S}$ is demiclosed on $\mathbb{C}$.

Lemma 6 (see [26]). Let $\left\{a_{n}\right\} \subset[0, \infty)$ be a sequence. Assume that $a_{n+1} \leq\left(1-\gamma_{n}\right) a_{n}+\delta_{n} \gamma_{n}$, where $\left\{\gamma_{n}\right\}$ is a sequence in $(0,1)$, and $\left\{\delta_{n}\right\}$ is a sequence satisfying $\sum_{n=1}^{\infty} \gamma_{n}=\infty$ and $\limsup _{n \rightarrow \infty} \delta_{n} \leq 0\left(\right.$ or $\left.\sum_{n=1}^{\infty}\left|\delta_{n} \gamma_{n}\right|<\infty\right)$. Then $\lim _{n \rightarrow \infty} a_{n}=$ 0 .

\section{Main Results}

In this section, we firstly present our problem and algorithm constructed. Consequently, we give the convergence analysis of the presented algorithm.

Problem 7. Let $\mathbb{C}$ be a nonempty closed convex subset of a real Hilbert space $\mathbb{H}$. Assume that

(1) $\Psi: \mathbb{C} \rightarrow \mathbb{C}$ is a weakly continuous and $\zeta$-strongly monotone mapping such that $R(\Psi)=\mathbb{C}$;

(2) $\mathbb{A}: \mathbb{C} \rightarrow \mathbb{H}$ is an $\varsigma$-inverse strongly $\Psi$-monotone mapping;

(3) $\varrho: \mathbb{C} \times \mathbb{C} \rightarrow \mathbb{R}$ is a bifunction satisfying conditions ( 1$)-(\mathfrak{F} 4)$ in Lemma 2.

Our objective is to

find $x^{\natural} \in \operatorname{VI}(\mathbb{A}, \Psi)$ such that $\Psi\left(x^{\natural}\right) \in \operatorname{EP}(\varrho, \mathbb{C})$.

We use $\Upsilon$ to denote the set of solutions of (8). In the following, we assume that $\Upsilon$ is nonempty. For solving Problem 7, we introduce the following algorithm.

Algorithm 8.

Step 0 (initialization). Let

$$
u_{0} \in \mathbb{C} \text {. }
$$


Step 1. For given $\left\{u_{n}\right\}$, let the sequence $\left\{v_{n}\right\}$ be generated iteratively by

$$
v_{n}=\operatorname{proj}_{\mathbb{C}}\left(\Psi\left(u_{n}\right)-\mu_{n} \mathbb{A} u_{n}\right), \quad n \geq 0,
$$

where $\operatorname{proj}_{\mathbb{C}}$ is the metric projection and $\left\{\mu_{n}\right\}$ is a real number sequence.

Step 2. For given $\left\{v_{n}\right\}$, find $\left\{z_{n}\right\}$ such that

$$
\varrho\left(z_{n}, y\right)+\frac{1}{\omega_{n}}\left\langle y-z_{n}, z_{n}-\left(1-\alpha_{n}\right) v_{n}\right\rangle \geq 0, \quad \forall y \in \mathbb{C},
$$

where $\left\{\omega_{n}\right\} \subset(0, \infty)$ and $\left\{\alpha_{n}\right\} \subset[0,1]$ are two real number sequences.

Step 3. For the previous sequences $\left\{u_{n}\right\}$ and $\left\{z_{n}\right\}$, let the $(n+$ 1)th sequence $\left\{u_{n+1}\right\}$ be generated by

$$
\Psi\left(u_{n+1}\right)=\kappa_{n} \Psi\left(u_{n}\right)+\left(1-\kappa_{n}\right) z_{n}, \quad n \geq 0,
$$

where $\left\{\kappa_{n}\right\} \subset[0,1]$ is a real number sequence.

Theorem 9. Assume that the following conditions are satisfied:

$$
\begin{aligned}
& \text { (C1) } \lim _{n \rightarrow \infty} \alpha_{n}=0 \text { and } \sum_{n} \alpha_{n}=\infty ; \\
& \text { (C2) } 0<\liminf _{n \rightarrow \infty} \kappa_{n} \leq \lim \sup _{n \rightarrow \infty} \kappa_{n}<1 ; \\
& \text { (C3) } \omega_{n} \in\left(\eta_{1}, \eta_{2}\right) \subset(0, \infty), \mu_{n} \in\left(\xi_{1}, \xi_{2}\right) \subset(0,2 \varsigma) \text {, }
\end{aligned}
$$
and $\zeta \in(0,2 \varsigma)$;

(C4) $\lim _{n \rightarrow \infty}\left(\mu_{n+1}-\mu_{n}\right)=0$ and $\lim _{n \rightarrow \infty}\left(\omega_{n+1}-\right.$ $\left.\omega_{n}\right)=0$.

Then the sequence $\left\{u_{n}\right\}$ generated by Algorithm 8 converges strongly to $x^{*} \in \Upsilon$.

Proof. Let $\breve{x} \in \Upsilon$. Hence $\breve{x} \in \mathrm{VI}(\mathbb{A}, \Psi)$ and $\Psi(\breve{x}) \in \mathrm{EP}(\varrho, \mathbb{C})$, noting that $\breve{x} \in \operatorname{VI}(\mathbb{A}, \Psi)$ implies $\Psi(\breve{x})=\operatorname{proj}_{\mathbb{C}}(\Psi(\breve{x})-v \mathbb{A} \breve{x})$ for all $\nu>0$. Hence $\Psi(\breve{x})=\operatorname{proj}_{\mathbb{C}}\left(\Psi(\breve{x})-\mu_{n} \mathbb{A} \breve{x}\right)$ for all $n \geq 0$. Thus, from (10), we have

$$
\begin{aligned}
\| v_{n}- & \Psi(\breve{x}) \|^{2} \\
= & \left\|\operatorname{proj}_{\mathbb{C}}\left(\Psi\left(u_{n}\right)-\mu_{n} \mathbb{A} u_{n}\right)-\operatorname{proj}_{\mathbb{C}}\left(\Psi(\breve{x})-\mu_{n} \mathbb{A} \breve{x}\right)\right\|^{2} \\
\leq & \left\|\left(\Psi\left(u_{n}\right)-\mu_{n} \mathbb{A} u_{n}\right)-\left(\Psi(\breve{x})-\mu_{n} \mathbb{A} \breve{x}\right)\right\|^{2} \\
= & \left\|\Psi\left(u_{n}\right)-\Psi(\breve{x})\right\|^{2}-2 \mu_{n}\left\langle\mathbb{A} u_{n}-\mathbb{A} \breve{x}, \Psi\left(u_{n}\right)-\Psi(\breve{x})\right\rangle \\
& +\mu_{n}^{2}\left\|\mathbb{A} u_{n}-\mathbb{A} \breve{x}\right\|^{2} \\
\leq & \left\|\Psi\left(u_{n}\right)-\Psi(\breve{x})\right\|^{2} \\
& -2 \mu_{n} \varsigma\left\|\mathbb{A} u_{n}-\mathbb{A} \breve{x}\right\|^{2}+\mu_{n}^{2}\left\|\mathbb{A} u_{n}-\mathbb{A} \breve{x}\right\|^{2} \\
\leq & \left\|\Psi\left(u_{n}\right)-\Psi(\breve{x})\right\|^{2}+\mu_{n}\left(\mu_{n}-2 \varsigma\right)\left\|\mathbb{A} u_{n}-\mathbb{A} \breve{x}\right\|^{2} .
\end{aligned}
$$

Condition (C3) and (13) imply that

$$
\left\|v_{n}-\Psi(\breve{x})\right\| \leq\left\|\Psi\left(u_{n}\right)-\Psi(\breve{x})\right\| .
$$

From Lemma 2 and (11), we get $z_{n}=F_{\omega_{n}}\left(1-\alpha_{n}\right) v_{n}$ for all $n \geq 0$. Since $\Psi(\breve{x}) \in \mathrm{EP}(\varrho, \mathbb{C})$, from Lemma 2 we deduce that $\Psi(\breve{x})=\digamma_{\Theta_{n}} \Psi(\breve{x})$ for all $n \geq 0$. So,

$$
\begin{aligned}
& \left\|z_{n}-\Psi(\breve{x})\right\| \\
& \quad=\left\|F_{\omega_{n}}\left(1-\alpha_{n}\right) v_{n}-F_{\Theta_{n}} \Psi(\breve{x})\right\| \\
& \quad \leq\left\|\left(1-\alpha_{n}\right) v_{n}-\Psi(\breve{x})\right\| \\
& \quad \leq\left(1-\alpha_{n}\right)\left\|v_{n}-\Psi(\breve{x})\right\|+\alpha_{n}\|\Psi(\breve{x})\|
\end{aligned}
$$

by (14)

$$
\leq\left(1-\alpha_{n}\right)\left\|\Psi\left(u_{n}\right)-\Psi(\breve{x})\right\|+\alpha_{n}\|\Psi(\breve{x})\| .
$$

It follows that

$$
\begin{aligned}
&\left\|\Psi\left(u_{n+1}\right)-\Psi(\breve{x})\right\| \\
& \leq \kappa_{n}\left\|\Psi\left(u_{n}\right)-\Psi(\breve{x})\right\|+\left(1-\kappa_{n}\right)\left\|z_{n}-\Psi(\breve{x})\right\| \\
& \leq \kappa_{n}\left\|\Psi\left(u_{n}\right)-\Psi(\breve{x})\right\| \\
&+\left(1-\kappa_{n}\right)\left(1-\alpha_{n}\right)\left\|\Psi\left(u_{n}\right)-\Psi(\breve{x})\right\| \\
&+\left(1-\kappa_{n}\right) \alpha_{n}\|\Psi(\breve{x})\| \\
&= {\left[1-\left(1-\kappa_{n}\right) \alpha_{n}\right]\left\|\Psi\left(u_{n}\right)-\Psi(\breve{x})\right\| } \\
&+\left(1-\kappa_{n}\right) \alpha_{n}\|\Psi(\breve{x})\| .
\end{aligned}
$$

By induction

$$
\left\|\Psi\left(u_{n}\right)-\Psi(\breve{x})\right\| \leq \max \left\{\left\|\Psi\left(u_{0}\right)-\Psi(\breve{x})\right\|,\|\Psi(\breve{x})\|\right\} .
$$

Hence, $\left\{\Psi\left(u_{n}\right)\right\}$ is bounded. Since $\Psi$ is $\zeta$-strongly monotone, we can get $\zeta\left\|u_{n}-\breve{x}\right\| \leq\left\|\Psi\left(u_{n}\right)-\Psi(\breve{x})\right\|$. So, $\left\|u_{n}-\breve{x}\right\| \leq$ $(1 / \zeta)\left\|\Psi\left(u_{n}\right)-\Psi(\breve{x})\right\| \leq(1 / \zeta) \max \left\{\left\|\Psi\left(u_{0}\right)-\Psi(\breve{x})\right\|,\|\Psi(\breve{x})\|\right\}$. This implies that $\left\{u_{n}\right\}$ is bounded. Next, we show $\| u_{n+1}-$ $u_{n} \| \rightarrow 0$. From $z_{n}=F_{\omega_{n}}\left(1-\alpha_{n}\right) v_{n}$, we have

$$
\begin{aligned}
& \left\|z_{n+1}-z_{n}\right\| \\
& =\left\|F_{\omega_{n+1}}\left(1-\alpha_{n+1}\right) v_{n+1}-F_{\omega_{n}}\left(1-\alpha_{n}\right) v_{n}\right\| \\
& \leq\left\|F_{\Phi_{n+1}}\left(1-\alpha_{n+1}\right) v_{n+1}-\digamma_{\Phi_{n+1}}\left(1-\alpha_{n}\right) v_{n}\right\| \\
& +\left\|F_{\omega_{n+1}}\left(1-\alpha_{n}\right) v_{n}-F_{\omega_{n}}\left(1-\alpha_{n}\right) v_{n}\right\| \\
& \leq\left\|\left(1-\alpha_{n+1}\right) v_{n+1}-\left(1-\alpha_{n}\right) v_{n}\right\| \\
& +\left\|F_{\omega_{n+1}}\left(1-\alpha_{n}\right) v_{n}-F_{\Theta_{n}}\left(1-\alpha_{n}\right) v_{n}\right\| .
\end{aligned}
$$


Using Lemma 3, we obtain

$$
\begin{aligned}
& \left\|F_{\Phi_{n+1}}\left(1-\alpha_{n}\right) v_{n}-F_{\Phi_{n}}\left(1-\alpha_{n}\right) v_{n}\right\|^{2} \\
& \leq \frac{\Phi_{n+1}-\Phi_{n}}{\Phi_{n+1}} \\
& \quad \times\left\langle F_{\omega_{n+1}}\left(1-\alpha_{n}\right) v_{n}\right. \\
& \quad-F_{\omega_{n}}\left(1-\alpha_{n}\right) v_{n}, F_{\omega_{n+1}}\left(1-\alpha_{n}\right) v_{n} \\
& \left.\quad-\left(1-\alpha_{n}\right) v_{n}\right\rangle \\
& \leq \frac{\left|\Phi_{n+1}-\Phi_{n}\right|}{\Phi_{n+1}}\left\|F_{\Phi_{n+1}}\left(1-\alpha_{n}\right) v_{n}-F_{\Phi_{n}}\left(1-\alpha_{n}\right) v_{n}\right\| \\
& \quad \times\left\|F_{\Phi_{n+1}}\left(1-\alpha_{n}\right) v_{n}-\left(1-\alpha_{n}\right) v_{n}\right\| .
\end{aligned}
$$

Then

$$
\begin{aligned}
& \left\|F_{\omega_{n+1}}\left(1-\alpha_{n}\right) v_{n}-F_{\omega_{n}}\left(1-\alpha_{n}\right) v_{n}\right\| \\
& \quad \leq \frac{\left|\Theta_{n+1}-\Theta_{n}\right|}{\Phi_{n+1}}\left\|F_{\Theta_{n+1}}\left(1-\alpha_{n}\right) v_{n}-\left(1-\alpha_{n}\right) v_{n}\right\| .
\end{aligned}
$$

By condition (C3), we have $\omega_{n}>\eta_{1}>0$. So,

$$
\begin{aligned}
\| z_{n+1}- & z_{n} \| \\
\leq & \left\|\left(1-\alpha_{n+1}\right) v_{n+1}-\left(1-\alpha_{n}\right) v_{n}\right\| \\
& +\frac{\left|\varrho_{n+1}-\Theta_{n}\right|}{\Phi_{n+1}} \\
& \times\left\|F_{\omega_{n+1}}\left(1-\alpha_{n}\right) v_{n}-\left(1-\alpha_{n}\right) v_{n}\right\| \\
\leq & \left(1-\alpha_{n+1}\right)\left\|v_{n+1}-v_{n}\right\| \\
& +\left|\alpha_{n+1}-\alpha_{n}\right|\left\|v_{n}\right\|+\frac{\left|\varrho_{n+1}-\varrho_{n}\right|}{\eta_{1}} \\
& \times\left\|F_{\Phi_{n+1}}\left(1-\alpha_{n}\right) v_{n}-\left(1-\alpha_{n}\right) v_{n}\right\| .
\end{aligned}
$$

From (10), we have

$$
\begin{aligned}
\| v_{n+1}- & v_{n} \| \\
= & \| \operatorname{proj}_{\mathbb{C}}\left(\Psi\left(u_{n+1}\right)-\mu_{n+1} \mathbb{A} u_{n+1}\right) \\
& -\operatorname{proj}_{\mathbb{C}}\left(\Psi\left(u_{n}\right)-\mu_{n} \mathbb{A} u_{n}\right) \| \\
\leq & \| \Psi\left(u_{n+1}\right)-\mu_{n+1} \mathbb{A} u_{n+1} \\
& -\left(\Psi\left(u_{n}\right)-\mu_{n+1} \mathbb{A} u_{n}\right)\left\|+\left|\mu_{n+1}-\mu_{n}\right|\right\| \mathbb{A}\left(u_{n}\right) \| \\
\leq & \left\|\Psi\left(u_{n+1}\right)-\Psi\left(u_{n}\right)\right\|+\left|\mu_{n+1}-\mu_{n}\right|\left\|\mathbb{A}\left(u_{n}\right)\right\| .
\end{aligned}
$$

Therefore,

$$
\begin{aligned}
& \left\|z_{n+1}-z_{n}\right\| \leq\left(1-\alpha_{n+1}\right)\left\|\Psi\left(u_{n+1}\right)-\Psi\left(u_{n}\right)\right\| \\
& \quad+\left|\alpha_{n+1}-\alpha_{n}\right|\left\|v_{n}\right\|+\left|\mu_{n+1}-\mu_{n}\right|\left\|\mathbb{A}\left(u_{n}\right)\right\| \\
& \quad+\frac{1}{\eta_{1}}\left|\varrho_{n+1}-\varrho_{n}\right|\left\|F_{\omega_{n+1}}\left(1-\alpha_{n}\right) v_{n}-\left(1-\alpha_{n}\right) v_{n}\right\| .
\end{aligned}
$$

It follows that

$$
\begin{aligned}
& \left\|z_{n+1}-z_{n}\right\|-\left\|\Psi\left(u_{n+1}\right)-\Psi\left(u_{n}\right)\right\| \\
& \quad \leq\left|\alpha_{n+1}-\alpha_{n}\right|\left\|v_{n}\right\|+\left|\mu_{n+1}-\mu_{n}\right|\left\|\mathbb{A}\left(u_{n}\right)\right\| \\
& \quad+\frac{1}{\eta_{1}}\left|\Theta_{n+1}-\varrho_{n}\right|\left\|F_{\Theta_{n+1}}\left(1-\alpha_{n}\right) v_{n}-\left(1-\alpha_{n}\right) v_{n}\right\| .
\end{aligned}
$$

Since $\lim _{n \rightarrow \infty} \alpha_{n}=0, \lim _{n \rightarrow \infty}\left(\mu_{n+1}-\mu_{n}\right)=0$, and $\lim _{n \rightarrow \infty}\left(\widehat{\omega}_{n+1}-\widehat{\omega}_{n}\right)=0$ and the sequences $\left\{\Psi\left(u_{n}\right)\right\},\left\{z_{n}\right\},\left\{v_{n}\right\}$, and $\left\{A u_{n}\right\}$ are bounded, we deduce that

$$
\limsup _{n \rightarrow \infty}\left(\left\|z_{n+1}-z_{n}\right\|-\left\|\Psi\left(u_{n+1}\right)-\Psi\left(u_{n}\right)\right\|\right) \leq 0
$$

Applying Lemma 4, we obtain

$$
\lim _{n \rightarrow \infty}\left\|z_{n}-\Psi\left(u_{n}\right)\right\|=0 .
$$

Thus,

$\lim _{n \rightarrow \infty}\left\|\Psi\left(u_{n+1}\right)-\Psi\left(u_{n}\right)\right\|=\lim _{n \rightarrow \infty}\left(1-\kappa_{n}\right)\left\|z_{n}-\Psi\left(u_{n}\right)\right\|=0$.

This together with the $\zeta$-strong monotonicity of $\Psi$ implies that

$$
\lim _{n \rightarrow \infty}\left\|u_{n+1}-u_{n}\right\|=0
$$

From (13) and (16), we derive

$$
\begin{aligned}
&\left\|\Psi\left(u_{n+1}\right)-\Psi(\breve{x})\right\|^{2} \\
& \leq \kappa_{n}\left\|\Psi\left(u_{n}\right)-\Psi(\breve{x})\right\|^{2}+\left(1-\kappa_{n}\right)\left\|z_{n}-\Psi(\breve{x})\right\|^{2} \\
& \leq \kappa_{n}\left\|\Psi\left(u_{n}\right)-\Psi(\breve{x})\right\|^{2}+\left(1-\kappa_{n}\right) \\
& \times\left[\left(1-\alpha_{n}\right)\left\|v_{n}-\Psi(\breve{x})\right\|^{2}+\alpha_{n}\|\Psi(\breve{x})\|^{2}\right]
\end{aligned}
$$




$$
\begin{aligned}
\leq & \left(1-\kappa_{n}\right) \\
\times & {\left[\left(1-\alpha_{n}\right)\left\|\left(\Psi\left(u_{n}\right)-\mu_{n} \mathbb{A} u_{n}\right)-\left(\Psi(\breve{x})-\mu_{n} \mathbb{A} \breve{x}\right)\right\|^{2}\right.} \\
& \left.+\alpha_{n}\|\Psi(\breve{x})\|^{2}\right]+\kappa_{n}\left\|\Psi\left(u_{n}\right)-\Psi(\breve{x})\right\|^{2} \\
\leq & \kappa_{n}\left\|\Psi\left(u_{n}\right)-\Psi(\breve{x})\right\|^{2}+\left(1-\kappa_{n}\right)\left(1-\alpha_{n}\right) \\
\quad \times & \left(\left\|\Psi\left(u_{n}\right)-\Psi(\breve{x})\right\|^{2}+\mu_{n}\left(\mu_{n}-2 \varsigma\right)\left\|A u_{n}-\mathbb{A} \breve{x}\right\|^{2}\right) \\
+ & \alpha_{n}\|\Psi(\breve{x})\|^{2} \\
\leq & \left\|\Psi\left(u_{n}\right)-\Psi(\breve{x})\right\|^{2} \\
& +\left(1-\kappa_{n}\right)\left(1-\alpha_{n}\right) \mu_{n}\left(\mu_{n}-2 \varsigma\right)\left\|A u_{n}-\mathbb{A} \breve{x}\right\|^{2} \\
& +\alpha_{n}\|\Psi(\breve{x})\|^{2} .
\end{aligned}
$$

Hence,

$$
\begin{aligned}
\left(1-\kappa_{n}\right) & \left(1-\alpha_{n}\right) \mu_{n}\left(2 \varsigma-\mu_{n}\right)\left\|\mathbb{A} u_{n}-\mathbb{A} \breve{x}\right\|^{2} \\
\leq & \left\|\Psi\left(u_{n}\right)-\Psi(\breve{x})\right\|^{2}-\left\|\Psi\left(u_{n+1}\right)-\Psi(\breve{x})\right\|^{2} \\
& +\alpha_{n}\|\Psi(\breve{x})\|^{2} \\
\leq & \left(\left\|\Psi\left(u_{n}\right)-\Psi(\breve{x})\right\|+\left\|\Psi\left(u_{n+1}\right)-\Psi(\breve{x})\right\|\right) \\
& \times\left\|\Psi\left(u_{n+1}\right)-\Psi\left(u_{n}\right)\right\|+\alpha_{n}\|\Psi(\breve{x})\|^{2} .
\end{aligned}
$$

Since $\alpha_{n} \rightarrow 0,\left\|\Psi\left(u_{n+1}\right)-\Psi\left(u_{n}\right)\right\| \rightarrow 0$, and $\liminf _{n \rightarrow \infty}(1-$ $\left.\kappa_{n}\right)\left(1-\alpha_{n}\right) \mu_{n}\left(2 \varsigma-\mu_{n}\right)>0$, we obtain

$$
\lim _{n \rightarrow \infty}\left\|A u_{n}-\mathbb{A} \ddot{x}\right\|=0 .
$$

Set $y_{n}=\Psi\left(u_{n}\right)-\mu_{n} \mathbb{A} u_{n}-\left(\Psi(\breve{x})-\mu_{n} \mathrm{~A} \breve{x}\right)$ for all $n$. By using the firm nonexpansivity of projection, we get

$$
\begin{aligned}
\| v_{n}- & \Psi(\breve{x}) \|^{2} \\
= & \| \operatorname{proj}_{\mathbb{C}}\left(\Psi\left(u_{n}\right)-\mu_{n} \mathbb{A} u_{n}\right)^{2} \\
& \quad-\operatorname{proj}_{\mathbb{C}}\left(\Psi(\breve{x})-\mu_{n} \mathbb{A} \breve{x}\right) \|^{2} \\
\leq & \left\langle y_{n}, v_{n}-\Psi(\breve{x})\right\rangle \\
= & \frac{1}{2}\left\{\left\|y_{n}\right\|^{2}+\left\|v_{n}-\Psi(\breve{x})\right\|^{2}-\left\|y_{n}-v_{n}+\Psi(\breve{x})\right\|^{2}\right\} \\
\leq & \frac{1}{2}\left\{\left\|\Psi\left(u_{n}\right)-\Psi(\breve{x})\right\|^{2}+\left\|v_{n}-\Psi(\breve{x})\right\|^{2}\right. \\
& \left.-\left\|\Psi\left(u_{n}\right)-v_{n}-\mu_{n}\left(\mathbb{A} u_{n}-\mathbb{A} \breve{x}\right)\right\|^{2}\right\} \\
= & \frac{1}{2}\left\{\left\|\Psi\left(u_{n}\right)-\Psi(\breve{x})\right\|^{2}+\left\|v_{n}-\Psi(\breve{x})\right\|^{2}\right. \\
& -\left\|\Psi\left(u_{n}\right)-v_{n}\right\|^{2}-\mu_{n}^{2}\left\|\mathbb{A} u_{n}-\mathbb{A} \breve{x}\right\| \\
& \left.+2 \mu_{n}\left\langle\Psi\left(u_{n}\right)-v_{n}, \mathbb{A} u_{n}-\mathbb{A} \breve{x}\right\rangle\right\} .
\end{aligned}
$$

It follows that

$$
\begin{aligned}
\left\|v_{n}-\Psi(\breve{x})\right\|^{2} \leq & \left\|\Psi\left(u_{n}\right)-\Psi(\breve{x})\right\|^{2}-\left\|\Psi\left(u_{n}\right)-v_{n}\right\|^{2} \\
& +2 \mu_{n}\left\|\Psi\left(u_{n}\right)-v_{n}\right\|\left\|\mathbb{A} u_{n}-\mathbb{A} \breve{x}\right\| .
\end{aligned}
$$

From (29) and (32), we have

$$
\begin{aligned}
&\left\|\Psi\left(u_{n+1}\right)-\Psi(\breve{x})\right\|^{2} \\
& \leq \kappa_{n}\left\|\Psi\left(u_{n}\right)-\Psi(\breve{x})\right\|^{2}+\left(1-\kappa_{n}\right) \\
& \quad \times\left[\left(1-\alpha_{n}\right)\left\|v_{n}-\Psi(\breve{x})\right\|^{2}+\alpha_{n}\|\Psi(\breve{x})\|^{2}\right] \\
& \leq \kappa_{n}\left\|\Psi\left(u_{n}\right)-\Psi(\breve{x})\right\|^{2}+\left(1-\alpha_{n}\right)\left(1-\kappa_{n}\right) \\
& \times\left\|\Psi\left(u_{n}\right)-\Psi(\breve{x})\right\|^{2}-\left(1-\kappa_{n}\right)\left\|\Psi\left(u_{n}\right)-v_{n}\right\|^{2} \\
&+\left(1-\kappa_{n}\right) \alpha_{n}\|\Psi(\breve{x})\|^{2}+2 \mu_{n}\left(1-\kappa_{n}\right) \\
& \times\left\|\Psi\left(u_{n}\right)-v_{n}\right\|\left\|\mathbb{A} u_{n}-\mathbb{A} \breve{x}\right\| \\
& \leq\left\|\Psi\left(u_{n}\right)-\Psi(\breve{x})\right\|^{2}-\left(1-\kappa_{n}\right)\left\|\Psi\left(u_{n}\right)-v_{n}\right\|^{2} \\
&+2 \mu_{n}\left\|\Psi\left(u_{n}\right)-v_{n}\right\|\left\|\mathbb{A} u_{n}-\mathbb{A} \breve{x}\right\|+\alpha_{n}\|\Psi(\breve{x})\|^{2} .
\end{aligned}
$$

Then, we obtain

$$
\begin{aligned}
\left(1-\kappa_{n}\right) & \left\|\Psi\left(u_{n}\right)-v_{n}\right\|^{2} \\
\leq & \left(\left\|\Psi\left(u_{n}\right)-\Psi(\breve{x})\right\|+\left\|\Psi\left(u_{n+1}\right)-\Psi(\breve{x})\right\|\right) \\
& \times\left\|\Psi\left(u_{n+1}\right)-\Psi\left(x_{n}\right)\right\| \\
& +2 \mu_{n}\left\|\Psi\left(u_{n}\right)-v_{n}\right\|\left\|\mathbb{A} u_{n}-\mathbb{A} \breve{x}\right\|+\alpha_{n}\|\Psi(\breve{x})\|^{2} .
\end{aligned}
$$

Since $\lim _{n \rightarrow \infty} \alpha_{n}=0, \lim _{n \rightarrow \infty}\left\|\Psi\left(u_{n+1}\right)-\Psi\left(u_{n}\right)\right\|=0$, and $\lim _{n \rightarrow \infty}\left\|\mathbb{A} u_{n}-\mathbb{A} \breve{x}\right\|=0$, we deduce that

$$
\lim _{n \rightarrow \infty}\left\|\Psi\left(u_{n}\right)-v_{n}\right\|=0 .
$$

Next, we prove $\lim \sup _{n \rightarrow \infty}\left\langle\Psi\left(x^{*}\right), v_{n}-\Psi\left(x^{*}\right)\right\rangle \geq 0$, where $x^{*}$ satisfies (GVI): $\left\langle\Psi\left(x^{*}\right), \Psi(x)-\Psi\left(x^{*}\right)\right\rangle \geq 0$, for all $x \in \Upsilon$ (note that $\Psi$ is $\zeta$-strongly monotone; we can easily deduce that the solution of (GVI) is unique). We take a subsequence $\left\{v_{n_{i}}\right\}$ of $\left\{v_{n}\right\}$ such that

$$
\begin{aligned}
\limsup _{n \rightarrow \infty} & \left\langle\Psi\left(x^{*}\right), v_{n}-\Psi\left(x^{*}\right)\right\rangle \\
& =\lim _{i \rightarrow \infty}\left\langle\Psi\left(x^{*}\right), v_{n_{i}}-\Psi\left(x^{*}\right)\right\rangle \\
& =\lim _{i \rightarrow \infty}\left\langle\Psi\left(x^{*}\right), \Psi\left(u_{n_{i}}\right)-\Psi\left(x^{*}\right)\right\rangle .
\end{aligned}
$$

By the boundedness of $\left\{u_{n_{i}}\right\}$, we can choose a subsequence $\left\{u_{n_{i j}}\right\}$ of $\left\{u_{n_{i}}\right\}$ such that $u_{n_{i_{j}}} \rightarrow z$ weakly. For the convenience, we may assume that $u_{n_{i}} \rightarrow z$. This implies that $\Psi\left(u_{n_{i}}\right) \rightarrow \Psi(z)$ due to the weak continuity of $\Psi$. Now, we show $z \in \Upsilon$. We firstly show $\Psi(z) \in \mathrm{EP}(\varrho, \mathbb{C})$. 
Note that $\Phi_{n} \in\left(\eta_{1}, \eta_{2}\right)$. Then we choose a subsequence $\left\{\Phi_{n_{i}}\right\}$ of $\left\{\Phi_{n}\right\}$ such that $\lim _{i \rightarrow \infty} \Phi_{n_{i}}=\emptyset \in\left(\eta_{1}, \eta_{2}\right)$. From (26) and (36), we deduce that $\left\|z_{n}-v_{n}\right\|=\left\|_{F_{\omega_{n}}}\left(1-\alpha_{n}\right) v_{n}-v_{n}\right\| \rightarrow 0$. Thus, $\left\|z_{n_{i}}-v_{n_{i}}\right\|=\left\|F_{\omega_{n_{i}}}\left(1-\alpha_{n_{i}}\right) v_{n_{i}}-v_{n_{i}}\right\| \stackrel{\rightarrow}{\rightarrow}$. From Lemma 2, we know that $F_{\varrho}$ is nonexpansive. By demiclosed principle (Lemma 5), we get immediately that $\Psi(z) \in \operatorname{Fix}\left(F_{\emptyset}\right)=$ $\mathrm{EP}(\varrho, \mathbb{C})$.

Next we prove $z \in \operatorname{VI}(\mathbb{A}, \Psi)$. Set

$$
R v= \begin{cases}A v+N_{C}(v), & v \in C, \\ \emptyset, & v \notin C .\end{cases}
$$

By [27], we know that $R$ is maximal $\Psi$-monotone. Let $(v, w) \in$ $G(R)$. Since $w-\mathbb{A} v \in N_{C}(v)$ and $u_{n} \in C$, we have $\langle\Psi(v)-$ $\left.\Psi\left(u_{n}\right), w-\mathbb{A} v\right\rangle \geq 0$. Noting that $v_{n}=\operatorname{proj}_{\mathbb{C}}\left(\Psi\left(u_{n}\right)-\mu_{n} \mathbb{A} u_{n}\right)$, we get

$$
\left\langle\Psi(v)-v_{n}, v_{n}-\left(\Psi\left(u_{n}\right)-\mu_{n} \AA u_{n}\right)\right\rangle \geq 0 .
$$

It follows that

$$
\left\langle\Psi(v)-v_{n}, \frac{v_{n}-\Psi\left(u_{n}\right)}{\mu_{n}}+\mathbb{A} u_{n}\right\rangle \geq 0 .
$$

Then,

$$
\begin{aligned}
& \left\langle\Psi(v)-\Psi\left(u_{n_{i}}\right), w\right\rangle \\
& \geq\left\langle\Psi(v)-\Psi\left(u_{n_{i}}\right), \mathbb{A} v\right\rangle \\
& \geq\left\langle\Psi(v)-\Psi\left(u_{n_{i}}\right), \mathbb{A} v\right\rangle \\
& -\left\langle\Psi(v)-v_{n_{i}}, \frac{v_{n_{i}}-\Psi\left(u_{n_{i}}\right)}{\mu_{n_{i}}}\right\rangle \\
& -\left\langle\Psi(v)-v_{n_{i}}, \mathbb{A} u_{n_{i}}\right\rangle \\
& =\left\langle\Psi(v)-\Psi\left(u_{n_{i}}\right), \mathbb{A} v-\mathbb{A} u_{n_{i}}\right\rangle \\
& +\left\langle\Psi(v)-\Psi\left(u_{n_{i}}\right), \mathbb{A} u_{n_{i}}\right\rangle \\
& -\left\langle\Psi(v)-v_{n_{i}}, \frac{v_{n_{i}}-\Psi\left(u_{n_{i}}\right)}{\mu_{n_{i}}}\right\rangle \\
& -\left\langle\Psi(v)-v_{n_{i}}, \mathbb{A} u_{n_{i}}\right\rangle \\
& \geq-\left\langle\Psi(v)-v_{n_{i}}, \frac{v_{n_{i}}-\Psi\left(u_{n_{i}}\right)}{\mu_{n_{i}}}\right\rangle \\
& -\left\langle\Psi\left(u_{n_{i}}\right)-v_{n_{i}}, \mathbb{A} u_{n_{i}}\right\rangle .
\end{aligned}
$$

Since $\left\|\Psi\left(u_{n_{i}}\right)-v_{n_{i}}\right\| \rightarrow 0$ and $\Psi\left(u_{n_{i}}\right) \rightarrow \Psi(z)$, we deduce that $\langle\Psi(v)-\Psi(z), w\rangle \geq 0$ by taking $i \rightarrow \infty$ in (41). Thus, $z \in R^{-1} 0$ by the maximal $\Psi$-monotonicity of $R$. Hence, $z \in \operatorname{VI}(\mathbb{A}, \Psi)$. Therefore, $z \in \Upsilon$. From (37), we obtain

$$
\begin{aligned}
\limsup _{n \rightarrow \infty} & \left\langle\Psi\left(x^{*}\right), v_{n}-\Psi\left(x^{*}\right)\right\rangle \\
= & \lim _{i \rightarrow \infty}\left\langle\Psi\left(x^{*}\right), \Psi\left(u_{n_{i}}\right)-\Psi\left(x^{*}\right)\right\rangle \\
= & \left\langle\Psi\left(x^{*}\right), \Psi(z)-\Psi\left(x^{*}\right)\right\rangle \geq 0 .
\end{aligned}
$$

From (12), we have

$$
\begin{aligned}
&\left\|\Psi\left(u_{n+1}\right)-\Psi\left(x^{*}\right)\right\|^{2} \\
& \leq \kappa_{n}\left\|\Psi\left(u_{n}\right)-\Psi\left(x^{*}\right)\right\|^{2} \\
&+\left(1-\kappa_{n}\right)\left\|\left(1-\alpha_{n}\right) v_{n}-\Psi\left(x^{*}\right)\right\|^{2} \\
& \leq \kappa_{n}\left\|\Psi\left(u_{n}\right)-\Psi\left(x^{*}\right)\right\|^{2}+\left(1-\kappa_{n}\right) \\
& \times {\left[\left(1-\alpha_{n}\right)\left\|v_{n}-\Psi\left(x^{*}\right)\right\|^{2}\right.} \\
&-2 \alpha_{n}\left(1-\alpha_{n}\right)\left\langle\Psi\left(x^{*}\right), v_{n}-\Psi\left(x^{*}\right)\right\rangle \\
&\left.+\alpha_{n}^{2}\left\|\Psi\left(x^{*}\right)\right\|^{2}\right] \\
& \leq \kappa_{n}\left\|\Psi\left(u_{n}\right)-\Psi\left(x^{*}\right)\right\|^{2}+\left(1-\kappa_{n}\right) \\
& \times {\left[\left(1-\alpha_{n}\right)\left\|\Psi\left(u_{n}\right)-\Psi\left(x^{*}\right)\right\|^{2}-2 \alpha_{n}\left(1-\alpha_{n}\right)\right.} \\
&\left.\times\left\langle\Psi\left(x^{*}\right), v_{n}-\Psi\left(x^{*}\right)\right\rangle+\alpha_{n}^{2}\left\|\Psi\left(x^{*}\right)\right\|^{2}\right] \\
&= {\left[1-\left(1-\kappa_{n}\right) \alpha_{n}\right]\left\|\Psi\left(u_{n}\right)-\Psi\left(x^{*}\right)\right\|^{2}+\left(1-\kappa_{n}\right) \alpha_{n} } \\
& \times \times\left\{2\left(1-\alpha_{n}\right)\left\langle-\Psi\left(x^{*}\right), v_{n}-\Psi\left(x^{*}\right)\right\rangle\right. \\
&\left.\quad+\alpha_{n}\left\|\Psi\left(x^{*}\right)\right\|^{2}\right\} .
\end{aligned}
$$

Using Lemma 6, we conclude that $\Psi\left(u_{n}\right) \rightarrow \Psi\left(x^{*}\right)$, and hence $u_{n} \rightarrow x^{*}$. This completes the proof.

\section{References}

[1] Y. Censor and T. Elfving, "A multiprojection algorithm using Bregman projections in a product space," Numerical Algorithms, vol. 8, no. 2, pp. 221-239, 1994.

[2] C. Byrne, "A unified treatment of some iterative algorithms in signal processing and image reconstruction," Inverse Problems, vol. 20, no. 1, pp. 103-120, 2004.

[3] Y. Censor, T. Bortfeld, B. Martin, and A. Trofimov, "A unified approach for inversion problems in intensity modulated radiation therapy," Physics in Medicine and Biology, vol. 51, pp. 23532365, 2006.

[4] Q. Yang, "The relaxed CQ algorithm solving the split feasibility problem," Inverse Problems, vol. 20, no. 4, pp. 1261-1266, 2004.

[5] B. Qu and N. Xiu, "A note on the CQ algorithm for the split feasibility problem," Inverse Problems, vol. 21, no. 5, pp. 1655$1665,2005$.

[6] H.-K. Xu, "Iterative methods for the split feasibility problem in infinite-dimensional Hilbert spaces," Inverse Problems, vol. 26, no. 10, Article ID 105018, 17 pages, 2010. 
[7] Y. Yao, Y.-C. Liou, and N. Shahzad, "A strongly convergent method for the split feasibility problem," Abstract and Applied Analysis, vol. 2012, Article ID 125046, 15 pages, 2012.

[8] L.-C. Ceng, Q. H. Ansari, and J.-C. Yao, "Relaxed extragradient methods for finding minimum-norm solutions of the split feasibility problem," Nonlinear Analysis: Theory, Methods \& Applications, vol. 75, no. 4, pp. 2116-2125, 2012.

[9] G. Stampacchia, "Formes bilinéaires coercitives sur les ensembles convexes," vol. 258, pp. 4413-4416, 1964.

[10] G. M. Korpelevič, "An extragradient method for finding saddle points and for other problems," Ėkonomika I Matematicheskie Metody, vol. 12, pp. 747-756, 1976.

[11] R. Glowinski, Numerical Methods for Nonlinear Variational Problems, Springer, New York, NY, USA, 1984.

[12] A. N. Iusem, "An iterative algorithm for the variational inequality problem," Computational and Applied Mathematics, vol. 13, pp. 103-114, 1994.

[13] M. A. Noor, "Some developments in general variational inequalities," Applied Mathematics and Computation, vol. 152, no. 1, pp. 199-277, 2004.

[14] A. Bnouhachem, M. Li, M. Khalfaoui, and Z. Sheng, "A modified inexact implicit method for mixed variational inequalities," Journal of Computational and Applied Mathematics, vol. 234, no. 12, pp. 3356-3365, 2010.

[15] A. Bnouhachem, M. A. Noor, and Z. Hao, "Some new extragradient iterative methods for variational inequalities," Nonlinear Analysis: Theory, Methods \& Applications, vol. 70, no. 3, pp. 13211329, 2009.

[16] A. Bnouhachem and M. A. Noor, "Inexact proximal point method for general variational inequalities," Journal of Mathematical Analysis and Applications, vol. 324, no. 2, pp. 1195-1212, 2006.

[17] A. Bnouhachem, M. A. Noor, and Th. M. Rassias, "Three-steps iterative algorithms for mixed variational inequalities," Applied Mathematics and Computation, vol. 183, no. 1, pp. 436-446, 2006.

[18] E. Blum and W. Oettli, "From optimization and variational inequalities to equilibrium problems," The Mathematics Student, vol. 63, pp. 123-145, 1994.

[19] P. L. Combettes and S. A. Hirstoaga, "Equilibrium programming in Hilbert spaces," Journal of Nonlinear and Convex Analysis, vol. 6, no. 1, pp. 117-136, 2005.

[20] M. A. Noor and W. Oettli, "On general nonlinear complementarity problems and quasi-equilibria," Le Matematiche, vol. 49, no. 2, pp. 313-331, 1994.

[21] Y. Yao, M. A. Noor, and Y.-C. Liou, "On iterative methods for equilibrium problems," Nonlinear Analysis: Theory, Methods \& Applications, vol. 70, no. 1, pp. 497-509, 2009.

[22] M. A. Noor, "Fundamentals of equilibrium problems," Mathematical Inequalities \& Applications, vol. 9, no. 3, pp. 529-566, 2006.

[23] S. Takahashi and W. Takahashi, "Strong convergence theorem for a generalized equilibrium problem and a nonexpansive mapping in a Hilbert space," Nonlinear Analysis: Theory, Methods \& Applications, vol. 69, no. 3, pp. 1025-1033, 2008.

[24] T. Suzuki, "Strong convergence theorems for infinite families of nonexpansive mappings in general Banach spaces," Fixed Point Theory and Applications, vol. 2005, no. 1, pp. 103-123, 2005.

[25] K. Goebel and W. A. Kirk, Topics in Metric Fixed Point Theory, vol. 28 of Cambridge Studies in Advanced Mathematics, Cambridge University Press, Cambridge, UK, 1990.
[26] H.-K. Xu, "Iterative algorithms for nonlinear operators," Journal of the London Mathematical Society, vol. 66, no. 1, pp. 240-256, 2002.

[27] L. J. Zhang, J. M. Chen, and Z. B. Hou, "Viscosity approximation methods for nonexpansive mappings and generalized variational inequalities," Acta Mathematica Sinica, vol. 53, no. 4, pp. 691-698, 2010. 


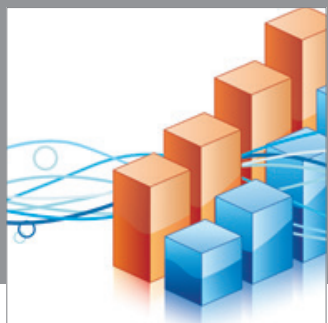

Advances in

Operations Research

mansans

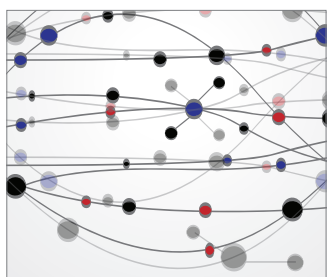

The Scientific World Journal
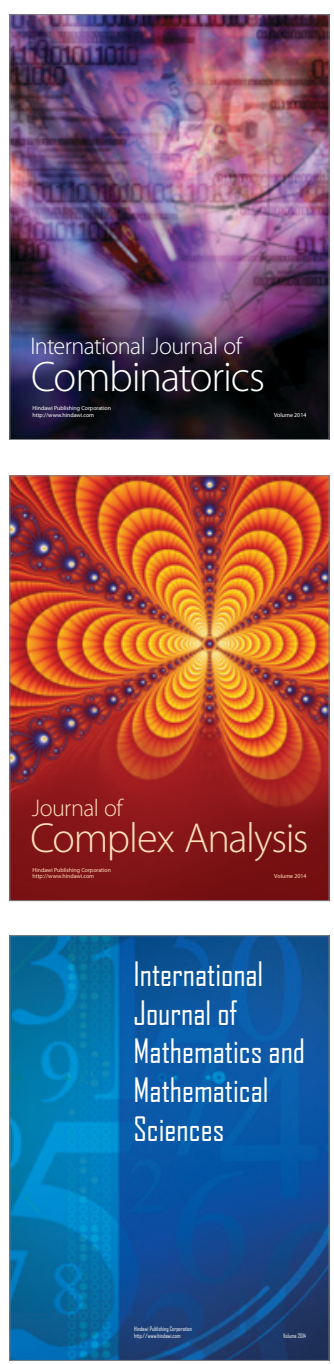
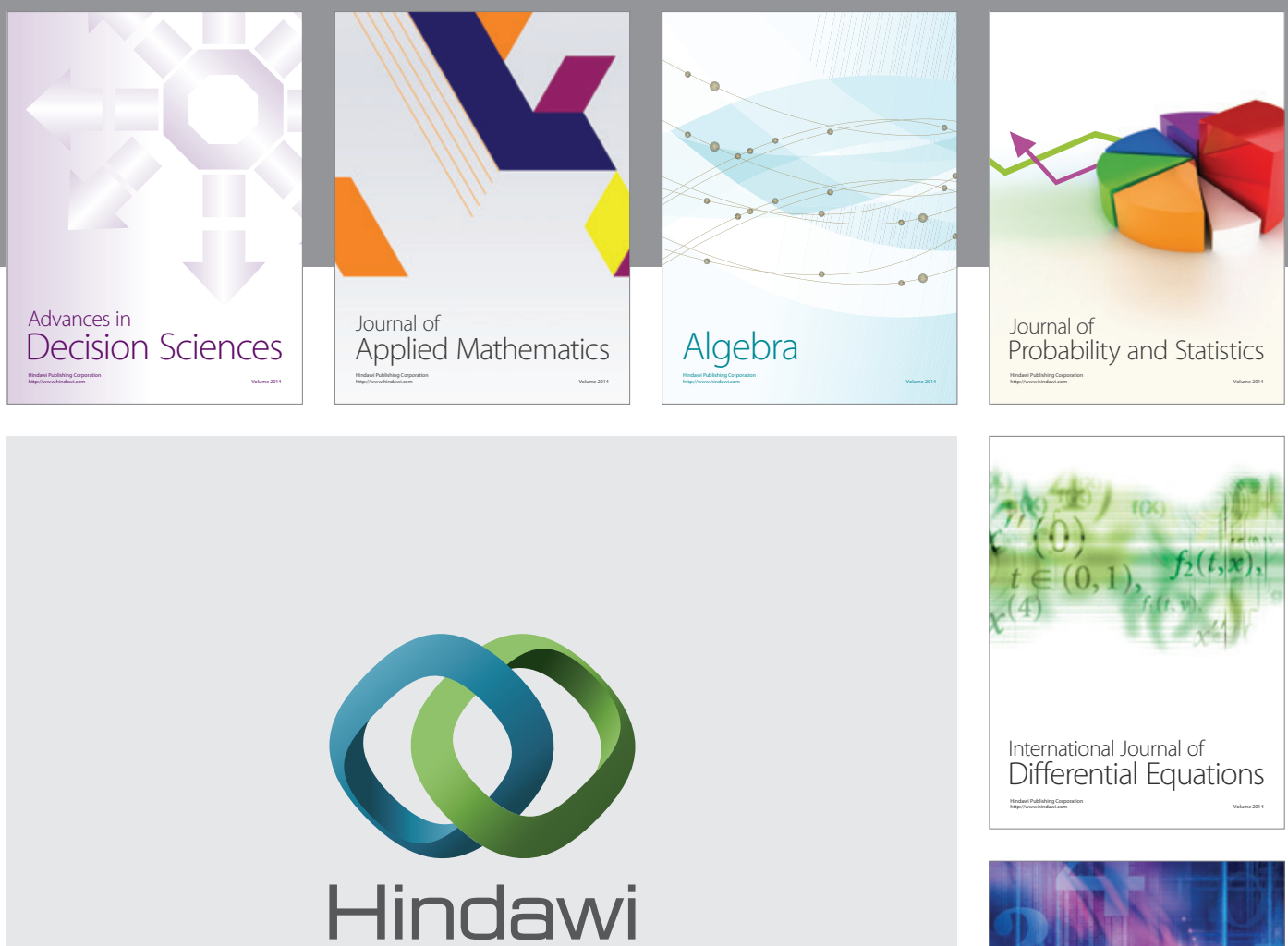

Submit your manuscripts at http://www.hindawi.com
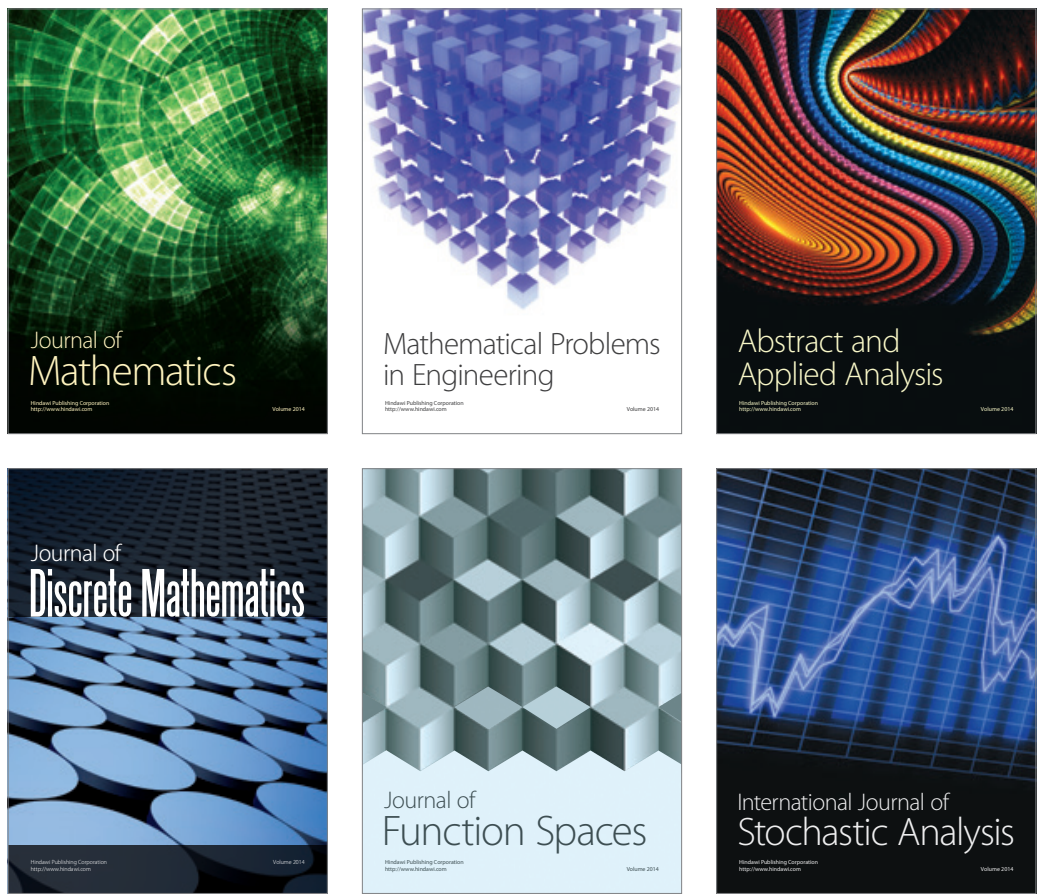

Journal of

Function Spaces

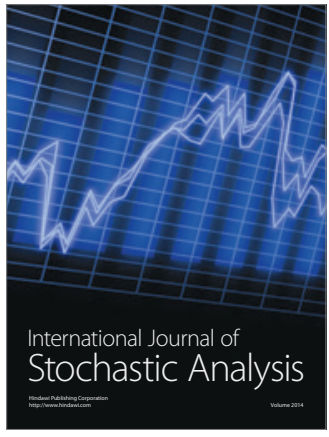

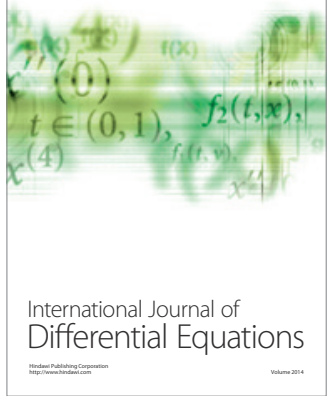
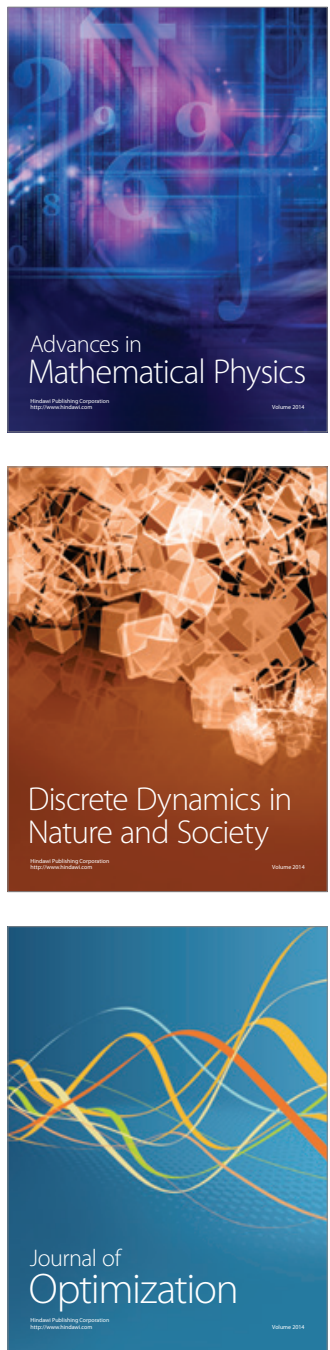